\title{
Recurrent erysipelas - risk factors and clinical presentation
}

\author{
Malin Inghammar ${ }^{1,2^{*}}$, Magnus Rasmussen ${ }^{1}$ and Adam Linder ${ }^{1,3}$
}

\begin{abstract}
Background: Erysipelas is a common infection that often recurs, but the impact of specific risk factors for reoccurrence remains elusive. In the present study we aimed at clarifying predisposing conditions for reoccurrence.

Methods: Medical records were reviewed from all patients $\geq 18$ years of age diagnosed with erysipelas at the Department of Infectious Diseases at Skåne University Hospital, Sweden, from January 2007 to February 2011. 502 patients were included, of which 357 were single episode erysipelas and 145 had recurrent erysipelas. These two groups were compared regarding underlying conditions and clinical presentation.

Results: Erysipelas in the lower limbs had the greatest propensity of recurrence. The associations between underlying conditions and recurrence were largely depending on the site of erysipelas. Overall, the most prominent risk factor for recurrence was lymphedema and other conditions causing a chronic impairment of the defence against microbes. Conditions temporarily disrupting the skin barrier (e.g. a local wound or toe web intertrigo), although likely being risk factors for erysipelas per se, did not seem to predispose to repeated episodes. Individuals with recurrent erysipelas tended to seek medical attention earlier, and were less likely to be hospitalized or receive intravenous antibiotics, but there was no evidence of any difference in inflammatory reaction when taking confounding factors into account.
\end{abstract}

Conclusions: In this large cross-sectional study of over 500 patients with erysipelas, lymphedema was the most prominent risk factors for recurrence although the distribution of predisposing conditions varies depending on the site of erysipelas.

Keywords: Erysipelas, Skin infection, Recurrent, Risk factor

\section{Background}

Erysipelas is a common infection of the superficial layer of the skin, in contrast to cellulitis and necrotizing fasciitis, which also involve the subcutaneous tissue. It has an estimated incidence of 19-24 per 10,000 inhabitants in European countries [1,2]. Erysipelas is defined as an acute onset of local signs of inflammation such as progressing erythema, associated with pain and swelling, clearly demarcated from the surrounding tissue. In the typical case erysipelas also manifests with systemic symptoms such as fever, chills and malaise and sometimes accompanied by nausea and vomiting $[3,4]$. The main causative agent is

\footnotetext{
* Correspondence: malin.inghammar@med.lu.se

'Department of Clinical Sciences, Division of Infection Medicine, Klinikgatan

1, Skåne University Hospital, Lund, Lund SE-221 85, Sweden

${ }^{2}$ The Division of Health Surveillance and Research, Statens Serum Institut, 5 Artillerivej, Copenhagen DK-2300, Denmark

Full list of author information is available at the end of the article
}

group A and group G $\beta$-hemolytic streptococci. Whether erysipelas also can be caused by Staphylococcus aureus or gram-negative bacteria is debated [5-7]. The most common site of the infection is the lower limb, accounting for about $80 \%$ of all cases [8]. In general erysipelas is a mild disease with low case-fatality, but a significant part of patients need in-hospital treatment [9]. The most common complication is recurrence, which occurs in $12-29 \%$ of the cases $[5,10,11]$.

There are various risk factors reported for erysipelas including disruption of the cutaneous barrier, venous insufficiency, lymphedema and overweight $[12,13]$. In the upper limbs, lymphedema or radical mastectomy are important risk factors [14], whereas risk factors for recurrence are less well defined [15]. Prophylaxis with penicillin $\mathrm{V}$ is sometimes recommended, but treatment criteria are not well defined. This common infection causes both 
suffering and medical expenses that should motivate appropriate prevention, which should be directed to patients with the greatest risk [1]. In this study, we hypothesized that patients with single episode erysipelas and patients with recurrent erysipelas differs regarding general characteristics and we aimed to clarify risk factors for erysipelas recurrence. We also aimed at assessing whether the clinical presentation in episodes of recurrent erysipelas differ from single episodes.

\section{Methods}

We identified all patients $\geq 18$ years of age, both hospitalized and outpatients, diagnosed with erysipelas at the Department of Infectious Diseases, Skåne University Hospital, Lund Sweden, between January 2007 and February 2011. The hospital is a university hospital serving 200,000 inhabitants, living in the city of Lund (southern Sweden) and surroundings. The diagnosis of erysipelas was determined by the admitting physician. The medical records were reviewed and data extracted according to a pre-specified protocol, all data was anonymized before analysis.

The patients were divided into two groups for comparison, single episode erysipelas (SE) and recurrent erysipelas (RE). SE was defined as patients with erysipelas without a history of previous episodes (medical records and anamnestic information). RE was defined as patients with more than one episode of erysipelas registered during the study period, or anamnestic information of previous episode outside the study period. Parameters are shown in the Additional file 1. Malignancy included both prior and present occurrence of all types. Previous regional operation was defined as surgical interventions at the area affected by erysipelas or adjacent locations. As a number of patients from the RE group was registered for more than one episode (separated by at least 30 days) during the study period, the medical record from the last recurrent episode that occurred within the study period was selected for comparison with the SE group.

The Ethics Committee of Lund University, Sweden (Dnr. 2011/674) approved of the study.

\section{Statistics}

Fisher's exact tests, Chi-squared tests and analysis of variance were used to assess the distribution of background factors in individuals with $\mathrm{SE}$ and RE, and to assess the distribution of markers of disease severity and clinical presentation between individuals with SE and RE. Multiple imputation was used to handle missing data, using chained equations with 20 imputation sets, see appendix for details $[16,17]$.

Logistic regression and exact logistic regression was used to assess predictors of recurrence. We considered gender, age (linear), localization (lower limbs, upper limbs, thorax/trunk, head/face) local operation (any kind), radiation therapy, severe obesity, liver disease, autoimmune/systemic inflammatory disease, immunosuppressive therapy, peripheral arterial insufficiency, peripheral venous insufficiency, lymphedema, wound, toe web intertrigo, diabetes mellitus, chronic obstructive pulmonary disease (COPD), cardiovascular disease, and polyneuropathy. Covariates assessed in univariable models were considered for inclusion in multivariable models at a $\mathrm{p}$-value of $<0.2$. Due to the anticipated modifying effect of erysipelas site, separate models were fitted for lower limbs, upper limbs, thorax and trunk, and head or face. Akaike's information criterion was used to assess model fits and Hosmer and Lemeshow's goodness-of-fit test in the complete case analyses was used to test the final models.

Logistic regression was used to assess whether individuals with recurrent episodes had a milder or more severe disease presentation, as measured by the need for hospitalization and/or initial intravenous antibiotics. Age, localization, time since onset of symptoms ( $<12$ hours, 13-72, >72) were included. Linear regression, adjusted for site and time since onset, was used to assess differences in C-reactive protein levels (CRP), (square root of CRP) and white blood cell counts (WBC), (log value of WBC). Likelihood ratio tests were used to test for differences and interactions. All analysis was performed with STATA/ SE (version 12.1; StataCorp LP, USA).

\section{Results}

\section{Patient demographics}

During the study period, 601 patients were registered with the diagnosis of erysipelas. Among them, 99 were excluded due to medical records that could not be retrieved, initial erysipelas diagnose that changed to other diagnose during the episode and non-acute patient visits.

A total of 573 episodes of erysipelas in 502 patients were identified during 2007-2012. The median age was 60 years (range 18-97), 58\% were male. The infection was located in the lower limbs in $69 \%$ of the episodes, in the upper limbs in $14 \%$, on the thorax or trunk in $8 \%$ and in the head or face in $9 \%$. The episodes were evenly distributed over the year, with no accumulation in either cold or warm months. Overall $64 \%$ received initial intravenous antibiotic treatment, and 55\% were hospitalized; 30\% had a positive local culture and $5 \%$ were bacteraemic.

Three hundred sixty-nine individuals had no previous history of erysipelas and 133 had a positive history. Fortysix individuals experienced at least one recurrence during the study period (median 3 episodes, range 2-5). Nine of these had no previous history but experienced recurrence during the study period.

\section{Clinical presentation and disease severity}

Individuals with SE, $\mathrm{n}=360$ (71\%), were compared with those with RE, $\mathrm{n}=142(29 \%)$. Neither age $(\mathrm{p}=0.06)$ nor 
the sex distribution $(\mathrm{p}=0.36)$ differed between the groups. Individuals with RE were more prone to seek medical care earlier, $33 \%<12$ hours since onset of symptoms vs. $17 \%$ in individuals with $\mathrm{SE}(\mathrm{p}=0.01)$, and were less likely to be hospitalized, adjusted odds ratio (aOR): 0.5 (95\% CI: $0.3-0.8$ ) or receive initial intravenous antibiotic treatment aOR: 0.6 (95\% CI: 0.4-0.9), estimated by logistic regression adjusted for age, localization, time since onset of symptoms and underlying co-morbidity. However, there was no difference in the distribution of the level of CRP ( $p=0.90)$ or white blood cell $(\mathrm{WBC})$ counts $(\mathrm{p}=0.58)$, estimated in linear regression models adjusted for time since onset and localization. In addition, there was no difference in the length of antibiotic treatment $(\mathrm{p}=0.78)$ nor in the length of stay (LOS) - analysed among those hospitalized $(\mathrm{n}=278)$, aOR for LOS $>7$ days for RE was $1.1(95 \%$ CI 0.5-2.3) adjusted for age, severe obesity, malignancy, COPD, diabetes and localization $(\mathrm{p}=0.93)$. See Table 1 for crude estimates.

\section{Risk factors for recurrent erysipelas}

The localization of erysipelas was associated with RE in univariable analyses, as were venous insufficiency,

Table 1 Clinical presentation in individuals with first and recurrent episodes of erysipelas

\begin{tabular}{|c|c|c|c|}
\hline & $\begin{array}{l}\begin{array}{l}\text { Single } \\
\text { episode }\end{array} \\
n=360 \\
(100 \%)\end{array}$ & $\begin{array}{l}\text { Recurrent } \\
\text { episodes } \\
n=142 \\
(100 \%)\end{array}$ & p-value \\
\hline Age, years (SD) & $58.0(18.7)$ & $61.5(16.1)$ & 0.05 \\
\hline Gender, $\%$ male & $211(58.6)$ & $75(52.8)$ & 0.24 \\
\hline Time since onset & & & $<0.01$ \\
\hline$<12$ hrs & $47(13.1)$ & $39(27.5)$ & \\
\hline $13-48 \mathrm{hrs}$ & $144(40.0)$ & $49(34.5)$ & \\
\hline$>48$ hrs & $85(23.6)$ & $29(20.4)$ & \\
\hline Missing & $84(23.3)$ & $25(17.6)$ & \\
\hline Localization $^{\text {1) }}$ & & & $<0.01$ \\
\hline Lower limbs & $231(64.2)$ & $111(78.2)$ & \\
\hline Upper limbs & $60(16.7)$ & 15 (10.6) & \\
\hline Thorax/trunk & $24(6.7)$ & $11(7.6)$ & \\
\hline Head/face & $45(12.5)$ & $6(3.5)$ & \\
\hline CRP, median (range) & $88(0-513)$ & $66(2-438)$ & 0.28 \\
\hline Missing & $57(15.8)$ & $29(20.4)$ & 0.22 \\
\hline WBC, median (range) & $11(2.7-68)$ & $13(2.6-28)$ & 0.82 \\
\hline Missing & $73(20.3)$ & $31(21.8)$ & 0.70 \\
\hline Inpatient treatment & $211(58.6)$ & $67(47.2)$ & 0.03 \\
\hline Initial iv antibiotics & $239(67.0)$ & $84(59.6)$ & 0.18 \\
\hline $\begin{array}{l}\text { Length of hospital } \\
\text { stay, median (range) }\end{array}$ & $5(1-34)$ & $5(1-22)$ & 0.61 \\
\hline
\end{tabular}

${ }^{1)}$-Global $p$-value from likelihood ratio test $<0.01$ ). lymphedema, severe obesity, previous operation and a history of malignancy, whereas a local wound and COPD was positively associated with SE, see Table 2 . As anticipated, there was a strong heterogeneity (i.e. effect modification) caused by the site of erysipelas.

In univariable analyses of erysipelas in the lower limbs $(\mathrm{n}=342)$ local factors, i.e. skin disease, venous insufficiency and lymphedema were predictive, see Table 3 . In multivariable analyses, there was statistical evidence for the association between RE and lymphedema, aOR 4.3 (95\% CI 1.3-14.0), venous insufficiency aOR 2.3 (95\% CI 1.0-5.2), skin disease aOR 1.9 (95\% CI 1.0-3.7) and COPD, OR 0.2 (95\% CI 0.04-1.0). However, effect estimates indicated a four-fold increased risk of recurrence for severe obesity and a two-fold for previous CABG, although these did not reach statistical significance.

In erysipelas located in the upper limbs $(n=75)$, gender, malignancy, local operation, lymphedema and local wound were predictive in univariable analyses. Due to the complex relationship between malignancy, operation and lymphedema, multivariable analyses were performed in three steps. In the first model, gender, malignancy and wound was included, in the second, operation and radiation was added, and thirdly lymphedema. The effect of gender in univariable analysis was completely confounded by a history of local operation. The final model included only operation and lymphedema. All of the effect of malignancy on the propensity for recurrence was mediated by a history local operation, aOR: 13.2 (95\% CI 2.0-85.4) which in turn was partly mediated by the presence of lymphedema, aOR: 7.4 (05\% CI 0.8-66.1). In univariable analyses, the OR for lymphedema (all patients with lymphedema had been operated on) was 43.5 (95\% CI: 7.5-249.7). Although analyses were based on small number, the OR for local operation, from stratified analysis in individuals without lymphedema, was 28.0 (95\% CI 3.3-236.4), which supports the idea that a local operation is a strong risk factor for recurrence and that the effect is enhanced by the development of lymphedema.

When erysipelas was located on thorax or the trunk $(\mathrm{n}=35)$, only malignancy, OR 10.0 (95\% CI 1.1-90.8), and wound, OR 0.09 (0.0-0.6), were associated with RE.

In erysipelas located in the head or face $(n=50)$, only $14 \%$ were $\mathrm{RE}$ and none of the examined covariates were associated with recurrence in univariable analyses, data not shown.

\section{Complete case analyses}

Missingness for CRP, WBC and time since onset of symptoms was highly dependent on hospitalization, but evenly distributed among individuals with RE and SE. The distributions of values from complete cases and imputed values in different strata of hospitalization were very similar. Estimates from additional analyses using 
Table 2 Distribution of underlying factors in individuals with single or recurrent episodes of erysipelas

\begin{tabular}{|c|c|c|c|c|}
\hline & $\begin{array}{l}\text { Single episode } \\
n=360(100 \%)\end{array}$ & $\begin{array}{l}\text { Recurrent episodes } \\
n=142(100 \%)\end{array}$ & Odds ratio $(95 \% \mathrm{Cl})$ & $\mathrm{p}$-value \\
\hline Age, years $(95 \% \mathrm{Cl})$ & $58.0(56.1-60.0)$ & $61.5(58.6-64.1)$ & $1.1(1.0-1.2)$ & 0.05 \\
\hline Gender, \% male & $211(58.6)$ & $75(52.8)$ & $0.8(0.5-1.2)$ & 0.36 \\
\hline Local operation & $71(19.7)$ & $46(32.4)$ & $2.0(1.3-3.0)$ & $<0.01$ \\
\hline$\left(A B G^{1)}\right.$ & $16(4.4)$ & $13(9.2)$ & $2.2(1.0-4.6)$ & 0.05 \\
\hline \multicolumn{5}{|l|}{ Localization } \\
\hline Lower limbs & $231(64.2)$ & $111(78.2)$ & 1 (reference) & \multirow[t]{4}{*}{$<0.01$} \\
\hline Upper limbs & $60(16.7)$ & $15(10.6)$ & $0.5(0.3-0.9)$ & \\
\hline Thorax/trunc & $24(6.7)$ & $11(7.6)$ & $0.9(0.4-2.0)$ & \\
\hline Head/face & $45(12.5)$ & $6(3.5)$ & $0.3(0.1-0.7)$ & \\
\hline Skin disease & $42(11.7)$ & $24(16.9)$ & $1.5(0.9-2.6)$ & 0.12 \\
\hline Malignancy & $53(14.7)$ & $36(25.4)$ & $2.0(1.2-3.7)$ & $<0.01$ \\
\hline Radiation therapy & $17(4.7)$ & $11(7.8)$ & $1.7(0.8-3.7)$ & 0.19 \\
\hline Immuno-suppressive therapy & $36(14.1)$ & $8(8.2)$ & $0.5(0.2-1.2)$ & 0.14 \\
\hline Arterial insufficiency & $13(3.6)$ & $8(5.6)$ & $1.6(0.6-3.9)$ & 0.32 \\
\hline Venous insufficiency ${ }^{1)}$ & $16(4.4)$ & $16(11.3)$ & $2.7(1.3-5.6)$ & $<0.01$ \\
\hline Lymphedema & $8(2.2)$ & $19(13.4)$ & $6.8(2.9-15.9)$ & $<0.01$ \\
\hline Diabetes mellitus & $52(14.4)$ & $25(17.6)$ & $1.3(0.8-2.1)$ & 0.38 \\
\hline Polyneuropathy & $11(3.1)$ & $4(2.8)$ & $0.9(0.3-2.9)$ & 0.89 \\
\hline Vascular disease & $62(17.2)$ & $24(16.9)$ & $1.0(0.6-1.6)$ & 0.93 \\
\hline COPD & $22(6.1)$ & $3(2.1)$ & $0.3(0.1-1.1)$ & 0.09 \\
\hline Systemic inflammatory disease & $16(4.5)$ & $4(2.8)$ & $0.6(0.2-1.8)$ & 0.41 \\
\hline Wound & $176(49.0)$ & $53(37.3)$ & $0.6(0.4-0.9)$ & 0.02 \\
\hline Toe web intertrigo ${ }^{1)}$ & $10(2.8)$ & $8(5.6)$ & $2.1(0.8-5.4)$ & 0.13 \\
\hline Severe obesitas & $1(0.3)$ & $4(2.8)$ & $10.4(1.2-93.9)$ & 0.04 \\
\hline Liver disease & $9(2.5)$ & $4(2.8)$ & $1.1(0.3-3.7)$ & 0.84 \\
\hline
\end{tabular}

1)-Calculated in the 342 individuals with erysipelas in the lower limbs, 229 and 113 respectively.

only complete cases yielded very little deviation from analyses including imputed values and did not change the conclusions drawn, see Additional file 1.

\section{Discussion}

In this large study of over 500 patients with erysipelas, we found lymphedema to be the most prominent risk factors for recurrence although the distribution of predisposing conditions varies depending on the site of erysipelas. In erysipelas in the head or face, none of the investigated factors were associated with recurrence.

There are only a few studies regarding risk factors for $\mathrm{RE}$, although previous results are generally in line with ours, most previous studies have neither been powered enough to allow for multivariable adjustments for coexisting risk factors nor to assess effect modification by the site of erysipelas [11,15,18-21]. Predisposing conditions for recurrent erysipelas located in the head or face has to the best of our knowledge not been studied previously. Our results show that there is a strong heterogeneity in the estimates from different sites of erysipelas, both in the propensity for recurrence and among the individual risk factors for recurrence, therefore analyses must be done separately for each site.

It is well known that women treated for breast cancer are at risk for ipsilateral erysipelas, especially if lymphedema develops $[14,22]$. Pavlotsky et al. found cardiac disease, malignancy and lymphedema to be associated with RE in the upper limbs in univariable analyses, but effect sizes are not given [18]. We found local operation and lymphedema to be strongly associated with RE, although it is difficult to obtain reliable estimates due to the complex relationship between tumor, surgery performed, radiation therapy, lymphedema and infection as outlined in Figure 1. In line with previous findings, we did not find evidence for radiation therapy to be a risk factor for recurrence [23].

Oddly, we found COPD to be protective against RE in the lower limbs even when adjusting for multiple covariates, this might be a spurious association or, more 
Table 3 Comparison between predisposing conditions of erysipelas in the lower limbs

\begin{tabular}{|c|c|c|c|c|c|c|}
\hline & $\begin{array}{l}\text { Single episode } \\
n=231(100 \%)\end{array}$ & $\begin{array}{l}\text { Recurrent episode } \\
n=111(100 \%)\end{array}$ & $\begin{array}{l}\text { OR }(95 \% \mathrm{Cl}) \text { from } \\
\text { univariable analysis } \\
(95 \% \mathrm{Cl})\end{array}$ & p-value & $\begin{array}{l}\text { OR }(95 \% \mathrm{Cl}) \text { from } \\
\text { multivariable analysis }{ }^{1)} \\
(95 \% \mathrm{Cl})\end{array}$ & p-value \\
\hline$\overline{C A B G}$ & $11(4.8)$ & $11(9.7)$ & $2.2(0.9-5.2)$ & 0.08 & $2.0(0.8-5.1)$ & 0.12 \\
\hline Skin disease & $26(11.3)$ & $23(20.7)$ & $2.1(1.1-3.8)$ & 0.02 & $1.9(1.0-3.7)$ & 0.05 \\
\hline Severe obesity & $1(0.4)$ & $4(3.6)$ & $8.5(0.9-77.9)$ & 0.06 & $4.6(0.5-45.8)$ & 0.19 \\
\hline Venous insufficiency & $14(6.1)$ & $16(14.4)$ & $2.6(1.2-5.6)$ & 0.02 & $2.3(1.0-5.2)$ & 0.05 \\
\hline Lymphedema & $6(2.6)$ & $9(8.1)$ & $3.3(1.1-9.5)$ & 0.03 & $4.3(1.3-14.0)$ & 0.02 \\
\hline Local wound & $118(51.1)$ & $49(44.1)$ & $0.8(0.5-1.2)$ & 0.23 & $0.8(0.5-1.3)$ & 0.42 \\
\hline COPD & $15(6.5)$ & $2(1.8)$ & $0.3(0.06-1.2)$ & 0.08 & $0.2(0.04-1.0)$ & 0.05 \\
\hline Diabetes mellitus & $44(19.1)$ & $20(18.0)$ & $0.9(0.5-1.7)$ & 0.82 & & \\
\hline Malignancy & $20(8.7)$ & $14(12.6)$ & $1.5(0.7-3.1)$ & 0.29 & & \\
\hline Toe web intertrigo & $10(4.3)$ & $8(7.2)$ & $1.7(0.7-4.5)$ & 0.27 & & \\
\hline Local operation²) & $49(21.2)$ & $27(24.3)$ & $1.2(0.7-2.0)$ & 0.28 & & \\
\hline
\end{tabular}

${ }^{1)}$-Adjusted for all of the variables in the column; ${ }^{2)}$ CABG included.

speculatively, due to a high use of antibiotics directed against airway pathogens in these patients which could reduce the colonizing burden of causative agents, e. $g$. group A streptococci.

It has been suggested that RE has a more severe disease presentation as measured by peak CRP and WBCs, length of fever and length of hospital stay [15]. However, these estimates were not adjusted for time since onset of symptoms or site of infection. We did not find evidence for differences in inflammatory parameters when taking time since onset of symptoms and potential confounders into account. Rerunning analyses, including only inpatient treated individuals, yielded similar results. It is possible that the lower propensity for hospitalization in patients with RE could be attributed to a higher threshold among

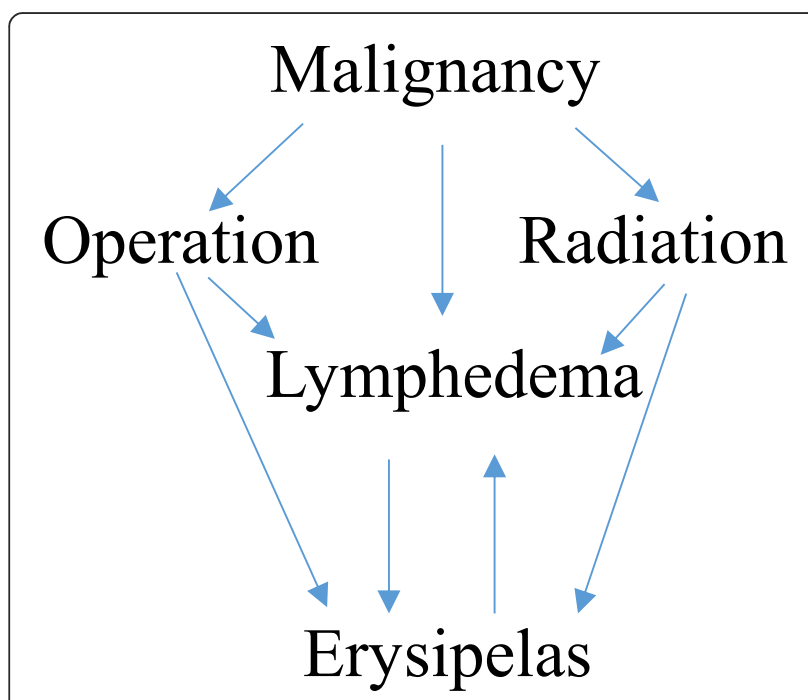

Figure 1 Directed acyclic graph (DAG) over relationship between predisposing conditions in erysipelas of the upper limbs. doctors for hospitalization and treatment with intravenous antibiotics, since patients with RE have experience of the disease.

This study has several strengths. It is one of the largest of its kind, which permits analyses of erysipelas per site of infection as well as adjustments for coexisting risk factors. We based our analyses on data collected from the medical charts, thus there was no risk of biased recall of information. Swedish health care is publicly financed and all inpatient care is provided independently of health insurance and the patient's financial status. A unique, lifelong ten-digit personal identity number assigned to each person living in Sweden provides the possibility of linking records in health care databases which limits the risk of biased selection. Erysipelas is by tradition cared for, at Skåne University Hospital, by specialists of infectious disease. We included all patients who were diagnosed with erysipelas at the Department of Infectious Diseases at Skåne University Hospital, during the study period.

There are also some limitations to this study. The retrospective design allow only available information restricted to medical records. Data were extracted according to a pre-specified protocol but some covariates may still be subject to interpersonal differences in the assessments.

The fraction of missing information on BMI was $81 \%$, why it was not considered reliable to impute values. Instead information on "severe obesity" was used, which was considered highly specific but with low sensitivity. If BMIvalues for all participants had been available, the point estimates for obesity (as defined by BMI-values), would probably have been statistically significant but diluted.

Patients were admitted to the Department of Infectious Diseases, although both as outpatient and inpatient, there is likely to be a bias towards patients with more severe disease. If the propensity to be admitted at the department of 
infectious disease is differential for patients with SE and RE, estimates could have been biased.

During the average two-year period of follow up, 3.3\% of the primarily naïve patients, experienced recurrence. Some of the patients classified as SE will probably later experience recurrence, this misclassification will bias estimates towards the null.

\section{Conclusion}

This study shows that a large proportion of cases with erysipelas are RE but these episodes do not seem to be more severe than SE. The most prominent risk factor for recurrence is lymphedema, regardless of the site of erysipelas, and other conditions causing a chronic impairment of the defence against microbes. Whereas conditions, temporarily disrupting the skin barrier (e.g. a local wound or toe web intertrigo), although being risk factors for erysipelas per se, did not seem to predispose to repeated episodes.

\section{Additional file}

Additional file 1: Appendix.

\section{Competing interests}

The authors declare that they have no competing interests.

\section{Authors' contributions}

Conception and design: MR, AL, Acquisition of data: EH, EK MR. Analysis and interpretation of data: MI, MR, AL. Drafting or revising the article: MI, MR, AL. Final approval of the manuscript: MI, MR, AL.

\section{Acknowledgement}

Eric Hultberg and Erik Kalin for help with data-extraction. Prof. Jonas Björk for invaluable statistical advice.

\section{Funding}

This work was supported by Swedish Government Research Grant (ALF) (AL MI and MR), Svenska läkarsällskapet Post-Doctoral grants (AL), CIHR IMPACT strategic training Post-Doctoral fellowship, UBC (AL), and the Swedish Research Council (VR) (AL).

\section{Author details}

${ }^{1}$ Department of Clinical Sciences, Division of Infection Medicine, Klinikgatan 1, Skåne University Hospital, Lund, Lund SE-221 85, Sweden. ${ }^{2}$ The Division of Health Surveillance and Research, Statens Serum Institut, 5 Artillerivej, Copenhagen DK-2300, Denmark. ${ }^{3}$ Division of Critical Care Medicine, Centre for Heart Lung Innovation, St. Paul's Hospital, University of British Columbia, 1081 Burrard Street, Vancouver, BC V6Z 1Y6, Canada.

Received: 11 November 2013 Accepted: 13 May 2014

Published: 18 May 2014

\section{References}

1. Goettsch WG, Bouwes Bavinck JN, Herings RM: Burden of illness of bacterial cellulitis and erysipelas of the leg in the Netherlands. JEADV 2006, 20(7):834-839.

2. Bartholomeeusen S, Vandenbroucke J, Truyers C, Buntinx F: Epidemiology and comorbidity of erysipelas in primary care. Dermatology 2007. 215(2):118-122.

3. Bisno AL, Stevens DL: Streptococcal infections of skin and soft tissues. N Engl J Med 1996, 334(4):240-245.
4. Bonnetblanc JM, Bedane C: Erysipelas: recognition and management. Am J Clin Dermatol 2003, 4(3):157-163.

5. Eriksson B, Jorup-Rönstrom C, Karkkonen K, Sjoblom AC, Holm SE: Erysipelas: clinical and bacteriologic spectrum and serological aspects. Clin Infect Dis 1996, 23(5):1091-1098.

6. Bernard P, Bedane C, Mounier M, Denis F, Catanzano G, Bonnetblanc JM: Streptococcal cause of erysipelas and cellulitis in adults: a microbiologic study using a direct immunofluorescence technique. Arch Dermatol 1989, 125(6):779-782.

7. Siljander T, Karppelin M, Vahakuopus S, Syrjanen J, Toropainen M, Kere J, Vuento R, Jussila T, Vuopio-Varkila J: Acute bacterial, nonnecrotizing cellulitis in Finland: microbiological findings. Clin Infec Dis 2008, 46(6):855-861.

8. Chartier C, Grosshans E: Erysipelas. Int J Dermatol 1990, 29(7):459-467.

9. Musette P, Benichou J, Noblesse I, Hellot MF, Carvalho P, Young P, Levesque $H$, Courtois H, Caron F, Lauret $P$, Joly P: Determinants of severity for superficial cellutitis (erysipelas) of the leg: a retrospective study. Eur J Intern Med 2004, 15(7):446-450.

10. Jorup-Rönstrom C: Epidemiological, bacteriological and complicating features of erysipelas. Scand J Infect Dis 1986, 18(6):519-524.

11. Jorup-Rönstrom C, Britton S: Recurrent erysipelas: predisposing factors and costs of prophylaxis. Infection 1987, 15(2):105-106.

12. Dupuy A, Benchikhi H, Roujeau JC, Bernard P, Vaillant L, Chosidow O, Sassolas B, Guillaume JC, Grob JJ, Bastuji-Garin S: Risk factors for erysipelas of the leg (cellulitis): case-control study. BMJ 1999, 318(7198):1591-1594.

13. Roujeau JC, Sigurgeirsson B, Korting HC, Kerl H, Paul C: Chronic dermatomycoses of the foot as risk factors for acute bacterial cellulitis of the leg: a case-control study. Dermatology 2004, 209(4):301-307.

14. Simon MS, Cody RL: Cellulitis after axillary lymph node dissection for carcinoma of the breast. Am J Med 1992, 93(5):543-548.

15. Karppelin M, Siljander T, Vuopio-Varkila J, Kere J, Huhtala H, Vuento R, Jussila T, Syrjanen J: Factors predisposing to acute and recurrent bacterial non-necrotizing cellulitis in hospitalized patients: a prospective case-control study. Clin Microbiol Infect 2010, 16(6):729-734.

16. Sterne JA, White IR, Carlin JB, Spratt M, Royston P, Kenward MG, Wood AM, Carpenter JR: Multiple imputation for missing data in epidemiological and clinical research: potential and pitfalls. BMJ 2009, 338:b2393.

17. Stuart EA, Azur M, Frangakis C, Leaf P: Multiple imputation with large data sets: a case study of the children's mental health initiative. Am J Epidemiol 2009, 169(9):1133-1139.

18. Pavlotsky F, Amrani S, Trau H: Recurrent erysipelas: risk factors. J Dtsch Dermatol Ges 2004, 2(2):89-95.

19. Cox NH: Oedema as a risk factor for multiple episodes of cellulitis/ erysipelas of the lower leg: a series with community follow-up. $\mathrm{Br}\rfloor$ Dermatol 2006, 155(5):947-950.

20. Lewis SD, Peter GS, Gomez-Marin O, Bisno AL: Risk factors for recurrent lower extremity cellulitis in a U.S. veterans medical center population. Am J Med Sci 2006, 332(6):304-307.

21. Leclerc S, Teixeira A, Mahe E, Descamps V, Crickx B, Chosidow O: Recurrent erysipelas: 47 cases. Dermatology 2007, 214(1):52-57.

22. Mozes M, Papa MZ, Karasik A, Reshef A, Adar R: The role of infection in post-mastectomy lymphedema. Surg Annu 1982, 14:73-83.

23. Lundstedt $D$, Gustafsson M, Malmström P, Johansson KA, Alsadius D, Sundberg A, Wilderang $U$, Holmberg E, Anderson H, Steineck G, Karlsson P. Symptoms 10-17 years after breast cancer radiotherapy data from the randomised SWEBCG91-RT trial. Radiother Oncol 2010, 97(2):281-287.

doi:10.1186/1471-2334-14-270

Cite this article as: Inghammar et al:: Recurrent erysipelas - risk factors and clinical presentation. BMC Infectious Diseases 2014 14:270. 